\title{
TUMOR DE WILMS DEL ADULTO
}

\author{
M. PASCUAL SAMANIEGO, J. CALLEJA ESCUDERO, T. ÁLVAREZ GAGO*, \\ V. GONZALO RODRÍGUEZ, C. MÜLLER ARTEAGA, E. FERNÁNDEZ DEL BUSTO
}

Servicio de Urología. *Servicio de Anatomía Patológica. Hospital Clínico Universitario de Valladolid. Valladolid.

Actas Urol Esp. 28 (7): 544-548, 2004

\section{RESUMEN}

TUMOR DE WILMS DEL ADULTO

El tumor de Wilms es una neoplasia renal maligna de origen embrionario de aparición excepcional en la edad adulta. Clínica y radiológicamente resulta indistinguible del carcinoma de células renales, por lo que su diagnóstico es anatomopatológico. Su estructura puede ser quística y debe ser considerado en el diagnóstico diferencial de la lesión quística renal.

El pronóstico en el adulto es peor que en la edad pediátrica debido a la mayor incidencia de recurrencia tumoral, a la falta de respuesta a los quimioterápicos clásicos utilizados tras la exéresis quirúrgica en niños y al estadío avanzado en el momento de la presentación clínica, como hallazgo casual de una masa abdominal asintomática en el 75\% de los casos.

Presentamos un nuevo caso de nefroblastoma del adulto en una mujer de 29 años, que debutó clínicamente como cólico nefrítico, con una configuración inicial ecográfica de masa renal quística con posterior evolución a lesión sólida.

Se carece por el momento de guías de tratamiento quimioterápico post-operatorio establecidas, pero algunos autores sugieren el empleo de carboplatino y etopósido debido a su comprobada eficacia en tumor de Wilms recurrente o refractario. Tras la administración de dichos fármacos, nuestra paciente permanece asintomática y sin recidiva tumoral 18 meses después de la cirugía.

PALABRAS CLAVE: Tumor de Wilms. Nefroblastoma. Adulto.

\section{ABSTRACT}

ADULT WILMS' TUMOR

Wilms' tumor is a malignant embryonic renal neoplasm that is exceptional in adults. There are not clinical data or radiographic investigations that can distinguish it from renal cell carcinoma. So the diagnostic is based in the pathological evaluation. It may be cystic and must be consider in the differential diagnosis of cystic lesions of the kidney.

The prognosis of Wilms' tumor in adults is worse than in children because of the high recurrence, the lower response rate to chemotherapy regimens and the advanced stage at the time of clinical presentation, like an asymptomatic abdominal mass in $75 \%$ of the cases.

We report a new case of nephroblastoma in a 29 years old woman presenting like a renal colic, with a cystic configuration by abdominal ultrasound initially, that changed into a solid renal mass later. There is not a definitive treatment protocol currently but some authors suggest a combination chemotherapy with carboplatin and etoposide because it is very effective in recurrent or refractory adult Wilms' tumor.

Our patient remains asymptomatic and without evidence of recurrence 18 months after the surgery.

KEY WORDS: Wilms's tumor. Nephroblastoma. Adult. 
$\mathrm{L}^{2}$ a presentación de un tumor de Wilms, característico de la infancia, en la edad adulta es muy infrecuente, suponiendo aproximadamente el $1 \%$ de todos los nefroblastomas ${ }^{1,2}$. Afecta por igual a hombres y mujeres con preferencia por la tercera década de la vida ${ }^{3}$. Su origen es embrionario, desarrollándose a partir del blastema metanéfrico primitivo ${ }^{1,4,5}$. La clínica y las pruebas complementarias radiológicas no permiten el diagnóstico diferencial con otras neoplasias rena$\mathrm{les}^{4}$. El criterio diagnóstico anatomopatológico exige la presencia de células blásticas y estructuras embrionarias tubulares o glomerulares sin evidencia de hipernefroma ${ }^{1,5,6}$.

El tratamiento clásico con cirugía radical, radioterapia según el estadio y quimioterapia, no ha tenido buenos resultados en el adulto, siendo peor su pronóstico respecto al niño, a pesar de su origen embrionario común y una histología microscópicamente idéntica ${ }^{6}$, por lo que se han propuesto múltiples protocolos quimioterápicos, no existiendo una guía de tratamiento bien establecido por el momento.

\section{CASO CLÍNICO}

Mujer de 29 años de edad, sin antecedentes patológicos de interés, que acudió inicialmente a la consulta de Urología por un cólico nefrítico derecho no complicado a la edad de 26 años, sin hematuria ni clínica miccional, presentando análisis de sangre y orina dentro de la normalidad. Se solicitó una ecografía abdominal en la que se apreciaba una imagen quística de $3 \mathrm{~cm}$ en el polo superior del riñón derecho (Fig. 1), con mala dife- renciación córtico-sinusal y alguna calcificación, pero sin dilatación de la vía urinaria.

Dos años después presentó nuevos episodios de cólico nefrítico derecho intermitente, por lo que se realizaron urografías intravenosas que informaban de imagen de desplazamiento y dilatación de los cálices medios y superiores del riñón derecho compatible con quistes renales y una litiasis radio-opaca en un cáliz mediorrenal derecho (Fig. 2). En este momento la ecografía de control (Fig. 1) evidenció una masa renal derecha sólida hiperecogénica de $10 \mathrm{~cm}$ de diámetro mayor, con alteración del contorno y del seno renal, calcificaciones puntiformes en su interior $\mathrm{y}$ caliectasia de los grupos medio $\mathrm{y}$ superior. El eco-doppler mostraba la vascularización interna de dicha masa renal, siendo el diagnóstico radiológico compatible con hipernefroma, sin poder descartar una pielonefritis xantogranulomatosa focal, dada la evolución de masa quística a sólida.

La TAC apoyaba el diagnóstico de hipernefroma derecho (Fig. 3), con adenopatías locorregionales inferiores a $5 \mathrm{~mm}$. La radiografía de tórax y la gammagrafía ósea no mostraban evidencia de metástasis.

Siendo el estudio preoperatorio normal, salvo una trombocitosis de 522000 plaquetas $/ \mathrm{mm}^{3}$, se realizó una nefrectomía radical derecha por vía subcostal transperitoneal.

El informe anatomopatológico describe macroscópicamente un riñón de 18 × 14 × $12 \mathrm{~cm}$, deformado por 7 nódulos confluentes de entre 1 y $7 \mathrm{~cm}$ de diámetro, bien delimitados por una

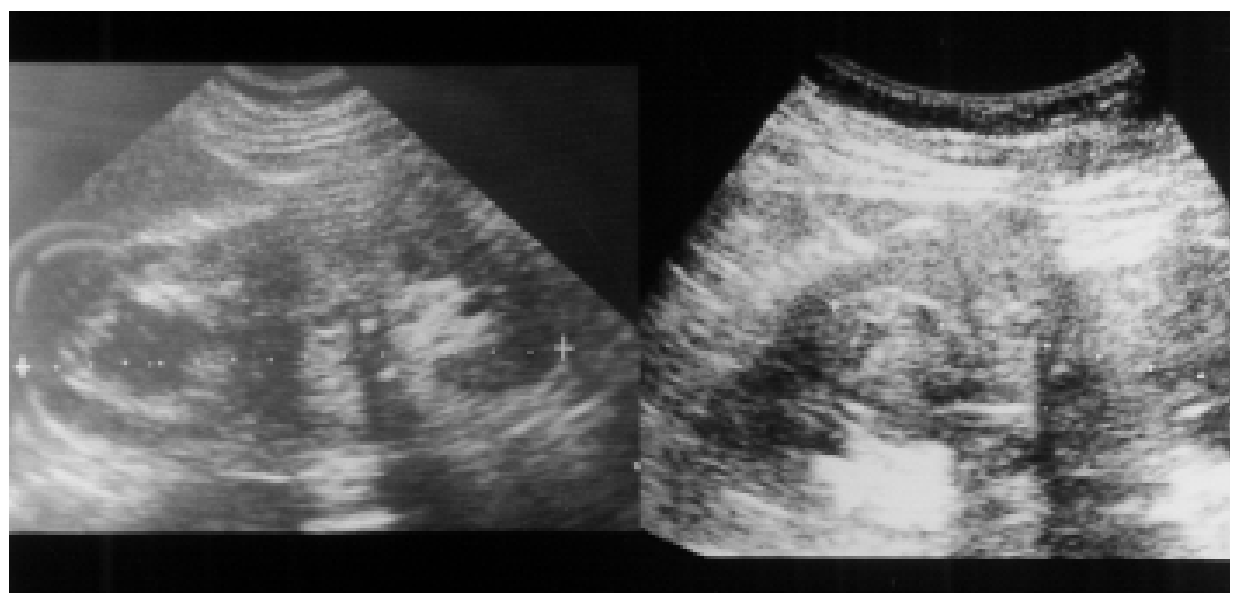

FIGURA 1. Derecha: ecografia renal derecha con imagen quistica polar superior de 3 cm de diámetro. Izquierda: ecografía de masa renal derecha sólida de $10 \mathrm{~cm} \mathrm{de}$ diámetro, que distorsiona el contorno y el seno renal. 


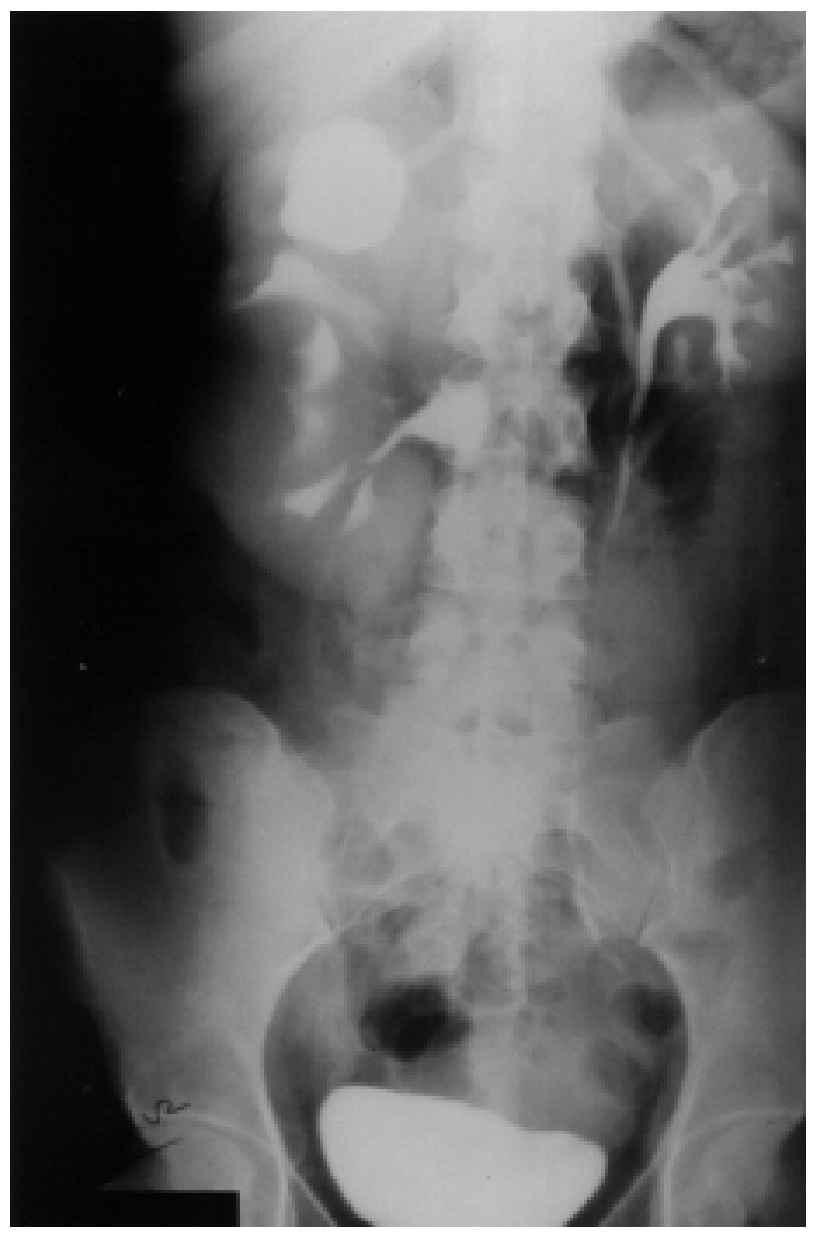

FIGURA 2. Urografia intravenosa. Masa renal polar superior derecha quistica que produce desplazamiento y ectasia de los cálices renales superiores.

pseudocápsula fibrosa de aspecto sarcomatoso, sin invasión de la cápsula ni de la vena renal. Sin embargo, el patólogo refleja la afectación tumoral en el cono de entrada de la arteria renal por la presencia de masa tumoral necrosada en su interior, comprobándose la existencia en la pared de dicha arteria, de múltiples placas de ateroma y de fibras elásticas. Microscópicamente, el tumor presentaba una proliferación neoplásica monofásica de células de estirpe blastomatosa pequeñas e hipercromáticas, en nidos sólidos con crecimiento en sábana y estructuras acinares y tubulares (Fig. 4).

Diagnóstico definitivo: tumor de Wilms (nefroblastoma) grado II de Fuhrman. Estadio I del NWTS (National Wilms Tumor Study).

Posteriormente la paciente fue remitida al Servicio de Oncología, donde recibió quimioterapia

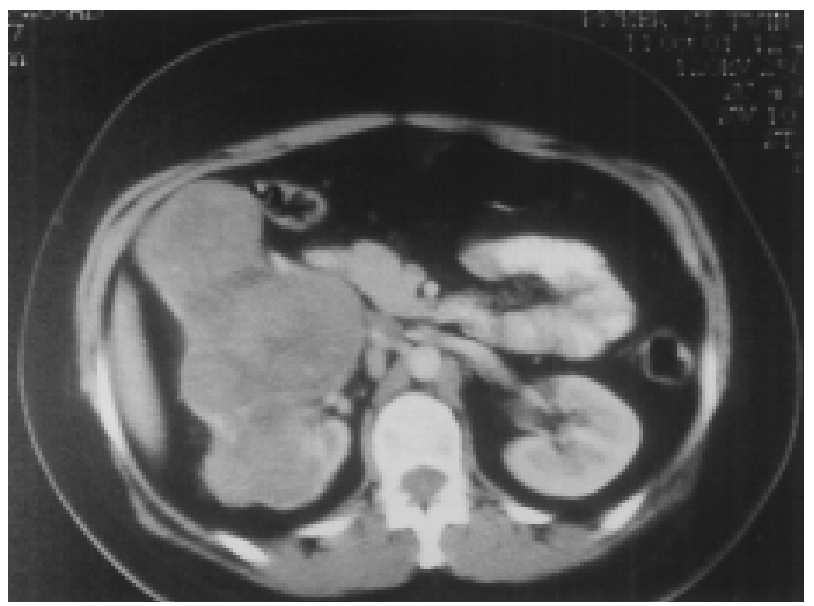

FIGURA 3. TAC. Masa renal derecha sólida de $10 \mathrm{~cm}$ de diámetro mayor.

en régimen de carboplatino y etopósido en tres ciclos a intervalos de 4 semanas. Después de año $\mathrm{y}$ medio desde la intervención, la paciente permanece asintomática y sin signos de recidiva tumoral ni enfermedad metastásica en la TAC de control.

\section{DISCUSIÓN}

Tan solo en torno al 1\% de los nefroblastomas se presentan en la edad adulta, siendo en estos pacientes muy difícil el diagnóstico diferencial previo a la cirugía, con el carcinoma de células renales, ya que no hay marcadores tumorales útiles y las pruebas radiológicas no aportan datos patognomónicos ${ }^{7}$.

La presentación clínica más frecuente es la de una masa abdominal asintomática de hallazgo incidental. Dentro de los sintomáticos, el dolor en el flanco es lo más común en el adulto. Kilton ${ }^{8}$ ya destacó que los pacientes que presentaban síntomas como dolor abdominal o hematuria, habian comenzado a manifestarse hasta en un $42 \%$ de los casos, más de un año antes de la detección del tumor. Nuestra paciente comenzó su clínica de cólico nefrítico intermitente casi tres años antes del diagnóstico definitivo.

Este mismo autor ya citado, presentó los siguientes criterios para el diagnóstico del tumor de Wilms del adulto:

- Paciente mayor de 15 años de edad, con neoplasia renal primaria en ausencia de elementos glandulares maduros de carcinoma de células renales. 


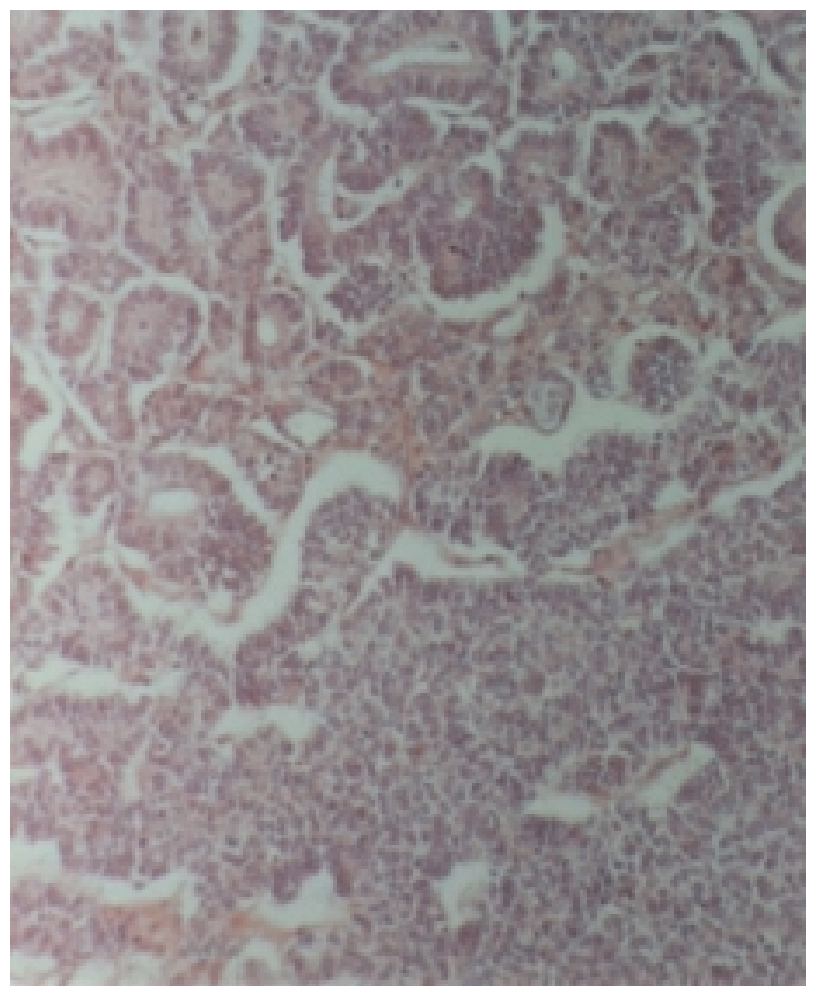

FIGURA 4. Estructuras tubulares y proliferación de células blásticas (HE X20).

- Confirmación histológica de componente celular primitivo blastematoso y estructuras embrionarias tubulares o glomerulares.

Esta neoplasia debe tenerse en cuenta en el diagnóstico diferencial de las lesiones quísticas renales ya que puede presentar esta configuración ${ }^{3}$, como ocurrió inicialmente en el caso presentado, aunque en los posteriores controles radiológicos evolucionó hacia una masa sólida. Así mismo el tumor presentaba pequeñas calcificaciones, hallazgo que se detecta en sólo un $10 \%$ de los $\operatorname{casos}^{2,3}$.

Se desaconseja la biopsia o punción percutánea diagnóstica del tumor como prevención de posibles metástasis y del riesgo aumentado de ruptura intraoperatoria que conlleva ${ }^{9}$, de acuerdo con las recomendaciones del NWTS (National Wilms Tumor Study) respecto a la realización de una nefrectomía radical antes de cualquier otra intervención ${ }^{7}$. La realización de una punción percutánea con aguja fina permite la clasificación tumoral en estadio I del NWTS- $5^{10}$. Sin embargo, la biopsia cerrada o abierta, supone la inclusión tumoral en el estadio II por la mayor probabilidad de diseminación.
El pronóstico depende fundamentalmente del estadio y de la histología, siendo peor en presencia de anaplasia $(5-6,4 \%)^{4}$. El estadio I del NWTS5 (asumiendo que dicho ensayo clínico se realizó a partir de pacientes menores de 16 años), considera el nefroblastoma limitado al riñón y completamente extirpado, sin afectación de la cápsula renal y sin invasión de los vasos venosos o linfáticos del seno renal, aceptando una mínima invasión de la grasa del mismo siempre que los márgenes de su zona media estén limpios de tumor. Beckwith $^{10}$ describe la presencia de células tumorales en la luz arterial como criterio de probable artefacto, argumentando el desplazamiento de las mismas durante la manipulación quirúrgica o patológica. Aunque nuestro patólogo comprobó la presencia de masa tumoral necrosada dentro de la luz arterial, los márgenes de lo que se ha denominado la zona media del seno renal, no se encontraban afectados. La evaluación de esta región es problemática y aún no está claramente definida. Lo que sí se ha considerado es la invasión de la vena renal y de los ganglios linfáticos como la principal vía de diseminación, siendo un criterio para el estadio II.

Por otra parte, la presencia de anaplasia se ha asociado a un aumento de la resistencia tumoral a la terapia adyuvante, siendo el factor histopatológico más determinante en el pronóstico, pero no sería un marcador de agresividad tumoral.

Comparativamente, el adulto con tumor de Wilms, tiene de forma significativa mucho peor pronóstico que el niño para cualquier estadio o histología ${ }^{3}$, habiendo estudios que reflejan sólo un $20 \%$ de pacientes libres de enfermedad a los tres años del tratamiento frente al $80 \%$ en la infancia ${ }^{11}$. Así mismo, el pronóstico es peor que el del carcinoma de células renales ${ }^{12}$. La extensión tumoral se produce por vía hemática y linfática, de modo que hasta un $25 \%$ de los adultos presentan metástasis en el momento del diagnóstico, sobre todo pulmonares, y cerca de un 30\% tienen afectación ganglionar regional ${ }^{3}$.

No se conoce la explicación para este peor pronóstico respecto al niño pero parece multifactorial ya que, a pesar de que la neoplasia se diagnostica en estadio III ó IV, en más de un 50\% de los adultos $^{4}$ las diferencias se mantienen cuando se compara por estadios ${ }^{5}$. Tampoco se ha aclarado si 
puede tener relación con un mayor porcentaje de adultos con tumor histológicamente desfavorable, puesto que no hay diferencias aparentes con el tumor infantil microscópicamente ${ }^{9}$, ni en su procedencia embriológica. Sin embargo, la anaplasia aparece con más frecuencia a partir de los tres años de edad ${ }^{13}$. También puede influir la mayor tolerancia de los niños a la quimioterapia intensiva.

El tratamiento multimodal consistente en nefrectomía radical con revisión del riñón contralateral, radioterapia y quimioterapia, a pesar de la mejora en la supervivencia a tres años, no ha logrado los resultados satisfactorios alcanzados en el nefroblastoma infantil ${ }^{6}$. La radioterapia del lecho tumoral es de dudosa efectividad en el adulto $^{1,2}$, no considerándose necesaria en el estadio $\mathrm{I}^{4,14}$. Sin embargo, la quimioterapia combinada, de superiores resultados a la utilización de fármacos aislados ${ }^{15}$, se emplea en todos los estadios aunque no hay un protocolo establecido, no habiéndose alcanzado buenos resultados con el empleo de drogas clásicamente utilizadas en el tumor infantil como la vincristina o la doxorrubicina $^{7}$. El sinergismo de la asociación de cisplatino y etopósido ha logrado respuestas favorables en el nefroblastoma del adulto recurrente o refractario a otros tratamientos como terapia de segunda línea, pero hay autores que han considerado su utilización como fármacos de primera elección ${ }^{16}$. Esto también ocurre en la combinación de carboplatino y etopósido, habiéndose empleado con éxito como terapia de rescate. Coincidiendo con la propuesta de otros autores ${ }^{6,17}$, aportamos una experiencia con buena respuesta a la administración de esta quimioterapia post-quirúrgica como primera línea en espera de futuras investigaciones que apoyen un protocolo de tratamiento de consolidación bien establecido.

\section{REFERENCIAS}

1. CARMONA CAMPOS E, ZARATE RODRÍGUEZ E.: Tumor de Wilms del adulto. Aportación de un caso. Actas Urol Esp 2001; 25 (10): 755-758.

2. SEGURA MARTÍN M, LORENZO ROMERO J, SALINAS SÁNCHEZ A y cols.: Tumor de Wilms del adulto. Descripción de un caso clínico. Arch Esp de Urol 1998; 51 (7): 705-708.
3. CAMCI C, TURK HM, ERKILIC S, BUYUKBERBER S, UNER A, OZSARAC O.: Early multimodal therapy in adult Wilms' tumor: case report. Journal of Chemotherapy 2002; 14 (5): 530-532.

4. CALVO RODRÍGUEZ M, FIRVIDA PÉREZ JL, VEGA VÁZQUEZ F y cols.: Tumor de Wilms del adulto. Arch Esp de Urol 2001; 54 (4): 370-374.

5. SING. VP, KANNAN N, LAKHTHAKIA R et al.: Adult Wilms' tumor (report of two cases). Indian J Pathol Microbiol 1997; 40 (3): 401-404.

6. BOZEMAN G, BISSADA NK, ABBOUD MR, LAVER J.: Adult Wilms' tumor: prognostic and management considerations. Urology 1995; 45 (6): 1055-1058.

7. ORDITURA M, DE VITA F, CATALANO G.: Adult Wilms' tumor. Cancer 1997; 80 (10): 1961-1965.

8. KILTON L, MATTHEWSMJ, COHEN MH.: Adult Wilms' tumor: a report of prolonged survival and review of literature. $J$ Urol 1980; 124: 1-5.

9. BABAIAN RJ, SKINNER DG, WAISMAN J.: Wilms' tumor in the adult patient. Cancer 1980; 45: 17131719.

10. BECKWITH JB.: National Wilms tumor study: an update for Pathologists. Pediatric and Development Pathology 1998; 1: 79-84.

11. PRESTIDGE BR, DONALDSON SS.: Treatment result among adults with chilhood tumors: a 20 years experience. Int J Radiat Oncol Biol Phys 1989; 17: $507-514$.

12. TAHRI A, BENCHEKROUN N, KARKOURI M et al.: Le néphroblastome de l'adulte. À propos de trois cas. Ann Urol 2001; 35: 257-261.

13. GREEN DM, TARBELL NJ, SHAMBERGER RC.: Solid tumors of childhood. In: De Vita, Lippincott, Philadelphia 1997: 2093-2097.

14. THOMAS PR.: Wilms' tumor: changing tole of radiation therapy. Semin Radiat Oncol 1997; 7 (3): 204211.

15. DAWSON NA, KLEIN MA, TAYLOR HG.: Salvage therapy in metastatic adult Wilms' tumor. Cancer 1988; 62 (5): 1017-1021.

16. ABRATT RP, DU PREEZ HM, KASCHULA R.: Adult Wilms' tumor: cisplatin and etoposide for relapse after adjuvant chemotherapy. Cancer 1990; 65: 890-892

17. PEIN F, TOURNADE MF, ZUCKER JM, BRUNATMENTIGNY $M$ et al.: Etoposide and carboplatin: a highly effective combination in relapsed or refractory Wilms' tumor. A phase II study by the French Society of Pediatric Oncology. J Clin Oncol 1994; 12 (5): 931-936.

Dr. M. Pascual Samaniego

C/ Miño, 5 - Esc. Dcha $2^{\circ} \mathrm{C}$

47140 Laguna de Duero (Valladolid)

Trabajo recibido el 30 septiembre de 2003) 